"Ovidius" University of Constanta, Faculty of Medicine

\begin{abstract}
:
A retrospective analysis of microbiology data from general infections was performed. From 105 isolates strains of Staphylococcus spp. $36(34,28 \%)$ were Staphylococcus epidermidis, 33 (31,42\%) Staphylococcus aureus, 21 (20\%) Staphylococcus haemolyticus, and 15 (14,28\%) were Staphylococcus hominis. Results: S. hominis isolates were predominantly resistant to betalactamins $(93,33 \%)$ and even Imidazole (60\%). 53,33\% of strains were resistent to aminosides and $33,33 \%$ to Ciprofloxacin. All strains (100\%) were sensitive to Vancomycin, but also all were susceptibile to Quinupristin-Dalfopristin. A high percentage of S. hominis were sensitive to Moxifloxacin, Linezolid $(93,33 \%)$, and to teicoplanin (86,67\%). Discussion: S. hominis is a member of skin normal flora, but all strains of S. hominis were isolated from generalized infection with a high rate of mortality.
\end{abstract}

Key words: Staphylococcus spp., Staphylococcus hominis, susceptibility, general infections.

\section{Voineagu Lavinia}

Faculty of Medicine, University Ovidius, Constanta lvoineagu@yahoo.com, tel 0722888975.

\section{Introduction}

Staphylococcus hominis (S. hominis) is a coagulase-negative member of the genus Staphylococcus. It occurs very commonly as a harmless commensal on human and animal skin. However, like many other coagulase-negative staphylococci, S. hominis may occasionally cause infection in patients whose immune systems are compromised.

S. hominis tends to colonize in areas with numerous apocrine glands, such as axillae and the pubic region. In a certain study, S. hominis was calculated to account for $22 \%$ of the total staphylococci species recovered from individuals, second to $\mathrm{S}$. epidermidis at $46 \%$. Most strains colonize on the skin for relatively short periods of time compared to other Staphylococcus species. Unlike S. epidermidis, S. hominis produces acid from trehalose, so the two tests together serve to identify the species [2].

There are cases of adult patients with sepsis caused by S. hominis. In literature mortality is written by $30 \%[4]$. 
Materials and methods.

This study determined Staphylococcus strains from hemocultures in general infections (Table I).

Table I Departments of hospital

\begin{tabular}{|l|l|}
\hline Department & No of samples \\
\hline Surgery & 3 \\
\hline Internal medcine & 6 \\
\hline Intensive care & 1 \\
\hline Pediatric intensive care & 3 \\
\hline Pediatrics & 2 \\
\hline
\end{tabular}

\section{METHOD:}

From January until July, 105 strains of Staphylococcus were isolated from hemocultures. Samples were collected and processed using standard microbiological protocols. The isolated strains were identified on device Vitek2 (Figure 1) on IDGP identification cards and the sensitivity test was perfomed on AST-P554 cards.
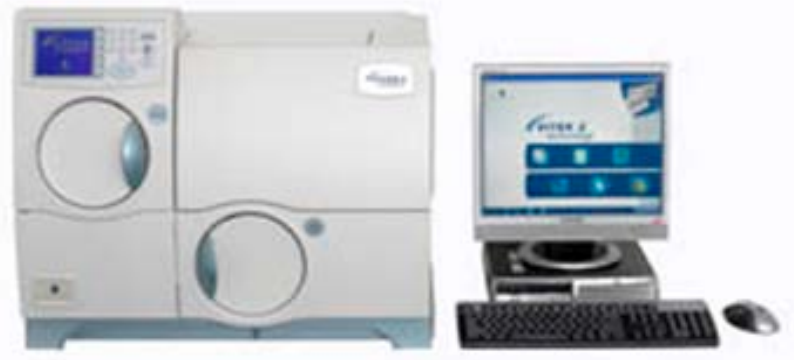

Figure 1 Vitek 2 system

\section{Results.}

From 105 isolates strains of Staphylococcus spp. 36 (34,28\%) were Staphylococcus epidermidis, $33(31,42 \%)$ Staphylococcus aureus, 21 (20\%) Staphylococcus haemolyticus, and 15 (14,28\%) were Staphylococcus hominis (Figure 2).

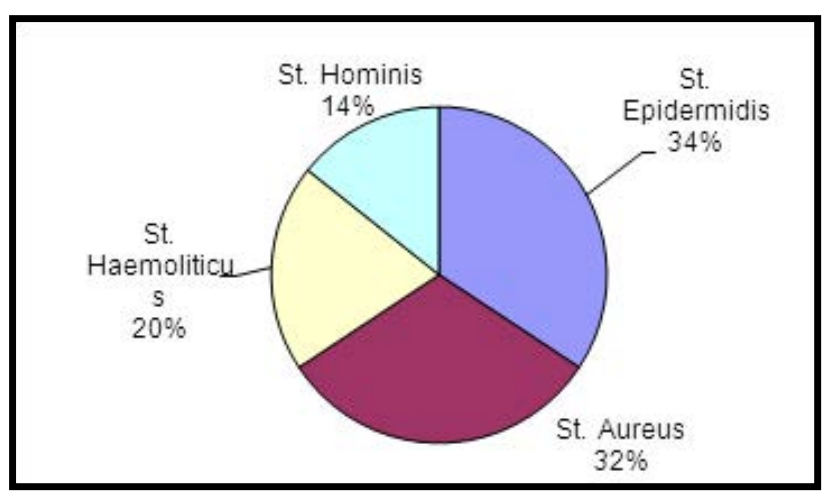

Figure 2 Percentage of Staphylococcus spp.

All isolates were from the hemoculture.

S. hominis isolates were predominantly resistant to betalactamins $(93,33 \%)$ and even Imidazole (60 $\%$ ). $53,33 \%$ of strains were resistent to aminosides and $33,33 \%$ to Ciprofloxacin. All strains (100\%) were sensitive to Vancomycin, but also all were susceptibile to Quinupristin-Dalfopristin. A high percentage of S. hominis were sensitive to Moxifloxacin, Linezolid $(93,33 \%)$, and to Teicoplanin $(86,67 \%)$.

\section{Discussion.}

Staphylococcus hominis is a member of skin normal flora, but all strains of $\mathrm{S}$. hominis were isolated from generalized infection. S. hominis infection frequency was appropriate literature data [5]. Results showed an increased resistance 
to bezilpeniciline, Imipenem, or Ciprofloxacin aminozide, and an increased number of Vancomycin, Quinupristin-Dalfopristin, Moxifloxacin, Linezolid and Teicoplanin - susceptible strains.

\section{References.}

1. Kloos, W. \& Schleifer, K. (1975). Isolation And Characterization Of Staphylococci From Human Skin. International Journal Of Systematic Bacteriology, 25, 62-79;

2. Fitzgibbon, J.E., Nahvi, M.D. \& John, J.F. (2010). A Sequence Variant Of Staphylococcus Hominis With A High Prevalence Of Oxacillin And Fluroquinolone Resistance. Research In Microbiology, 152, 805-810;
3. Palazzo, I.C.V., d'Azevedo, P.A., Secchi, C. \& Pignatari, A.C.C. (2008). Staphylococcus Hominis Subsp. Novobiosepticus Strains Causing Nosomical Bloodstream Infection In Brazil. Journal Of Antimicrobial Chemotherapy, 62, 1222-1226;

4. Chavez, F., Alvarez, M.G., Sanz, F., Alba, C. \& Joaquin, R.O. (2005). Nosocomical Spread Of Staphylococcus Hominis Subsp. Novobiosepticus Strain Causing Sepsis In A Neonatal Intesive Care Unit. Journal Of Clinical Microbiology, 43, 4877-4879;

5. Conde Garcia, R. Seisdedos Elcuaz, E. Jerez Fernandez, R. Ruiz Martin De La Torre, S. Canales Ugarte, T. (2012). General And Risk Management, Patient Safety (Including: Medication Errors, Quality Control) Analysis Of Infections Associated With Central Vascular Catheters Used For Parenteral Nutrition Administration Eur $J$ Hosppharm;19:100 DOI:10.1136/Ejhpharm-2012-000074.41. 